\title{
POLARISATION EFFECTS IN LIDAR SIGNALS: DETERMINATION, CORRECTION, AND UNCERTAINTY CALCULATION
}

\author{
Volker Freudenthaler ${ }^{1 *}$
}

\section{${ }^{I}$ Fakultät für Physik, Meteorologisches Institut, Ludwig-Maximilians-Universität, 80333 München, Theresienstrasse 37, Germany,*volker.freudenthaler@lmu.de}

\begin{abstract}
An analytical framework, based on the MüllerStokes formalism, is presented, which enables us to determine the impact of polarisation effects of the lidar optics on the lidar signals and on the measured atmospheric linear depolarisation. Furthermore, the equations enable us to investigate the accuracy of the gain ratio calibration of depolarisation channels with different calibration setups, to correct some of the polarisation effects, and to perform uncertainty analyses.
\end{abstract}

\section{INTRODUCTION}

The purpose of atmospheric depolarisation measurements with lidar is mainly to discern between more or less depolarising scatterers. In order to increase the accuracy, we have to analyse the impact of potential error sources, which is only possible for such sources that are included in the underlying analytical model of the lidar. While the consideration of errors due to random signal noise is largely standard, systematic errors in lidar signals very often have been neglected in the past or were included only for one or few parameters, supposedly because a complete mathematical model contains too many and often unknown parameters. This presentation gives an introduction to the mathematical lidar model recently published [1] to approach this problem regarding the polarization dependent systematic errors. For references or more detailed explanations please refer to this open access paper.

Polarization related systematic errors arise in lidar signals by three reasons: an uncertain state of polarization of the emitted laser beam, optical elements with polarization effects, and an inaccurate calibration of the gain ratio of the two lidar channels. The lidar model shown in Fig. 1 comprises all those parts, and at the same time it tries to reduce the number of parameters by combining the optics in three essential modules, which are the emitter optics $\left(\mathbf{M}_{E}\right)$, the receiver optics $\left(\mathbf{M}_{O}\right)$, and the polarization analysing optics $\left(\mathbf{M}_{T, R}\right)$ that includes a transmitted $(T)$ and a reflected $\left.{ }_{R}\right)$ path of the polarizing beam splitter (PBS) with the subsequent optical elements.

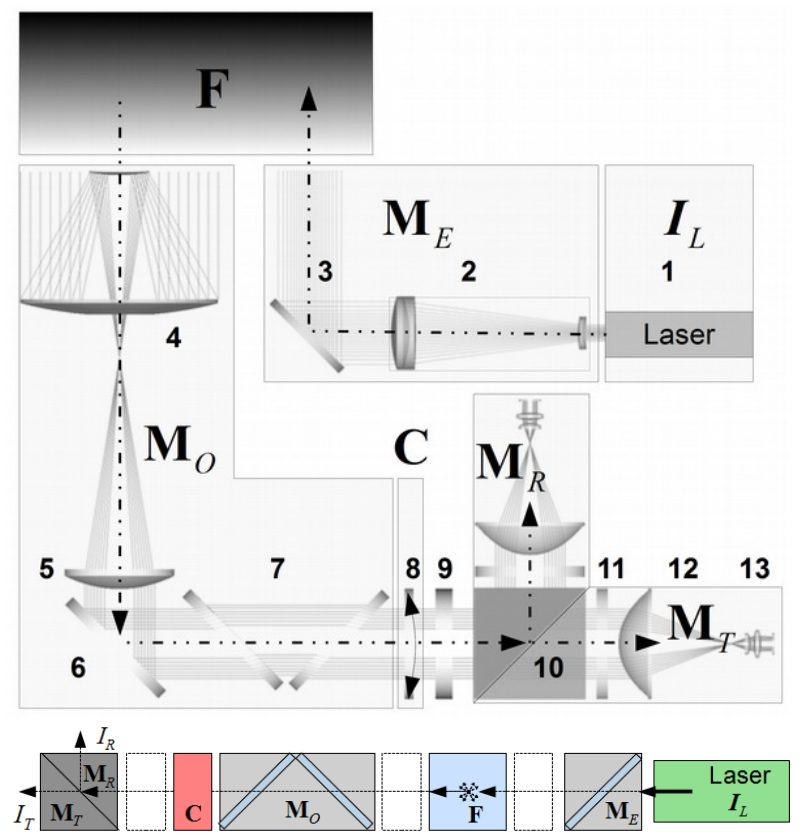

Figure 1 Top: Exemplary depolarisation lidar setup with laser 1, beam expander 2, steering mirror 3, receiving telescope 4, collimator 5, folding mirror 6 , dichroic beam-splitters 7 , a rotating element for polarisation calibration 8 , interference filter 9 , and polarising beam-splitter cube 10 (PBS, polarising beam-splitter). The neutral density filters and cleaning polarisers 11, detector optics 12, and the detectors 13 .

The system can be subdivided in functional blocks which can be described with the Stokes-Müller formalism: $\boldsymbol{I}_{L}$ is the Stokes vector of the laser source, $\mathbf{M}_{E}$ is the Müller matrix of the the laser emitter optics, $\mathbf{F}$ of the atmospheric backscattering volume including depolarisation, $\mathbf{M}_{O}$ includes receiver optics as beamsplitters, $\mathbf{C}$ is the calibrator, and $\mathbf{M}_{T, R}$ is the polarising beam-splitter including the detector optics for the transmitted $(T)$ and reflected $(R)$ optical branches. Bottom: simplified schematic of the setup. 
This model cannot comprise all possible aerosol lidar systems but, we believe, a majority. Each module is described by a Müller matrix of a rotated retarding diattenuator, which is defined by the linear diattenuation parameter $D$, the retardance $\Delta$, and the rotation $\theta$ of the module around the optical axis. The state of polarization of the emitted laser beam and the output beams in the transmitted and reflected channel are described by Stokes vectors $\boldsymbol{I}_{L}$ and $\boldsymbol{I}_{\boldsymbol{T}, \boldsymbol{R}}$, respectively, which are related by Eq. (1). The gain factors $\eta_{T, R}$ include the attenuation of optics and filters behind the PBS and the electronic amplification of each channel ${ }_{T, R}$.

$$
\boldsymbol{I}_{T, R}=\eta_{T, R} \mathbf{M}_{T, R} \mathbf{C} \mathbf{M}_{O} \mathbf{F} \mathbf{M}_{E} \boldsymbol{I}_{L}
$$

The equations for both signal intensities $I_{T, R}$, which is the first component of the Stokes vector, can be combined in one equation $I_{s}$ :

$$
I_{S}=\eta_{S}\left\langle\mathbf{A}_{S}|\mathbf{C}| I_{\text {in }}\right\rangle ; \text { with } S \in\{T, R\}
$$

The descriptions of the analyser part $<\boldsymbol{A}_{\boldsymbol{S}} \mid$ and of the input Stokes vector $\mid \boldsymbol{I}_{T, R}>$ are the same for all calibrators $\mathbf{C}$ (see below) and also for the standard signals without calibrator, wherefore we have to develop them only once. The atmospheric depolarisation is described by the Müller matrix for randomly oriented aerosol [2] as in Eq.(3)

$$
\mathbf{F}=F_{11}\left(\begin{array}{cccc}
1 & 0 & 0 & 0 \\
0 & a & 0 & 0 \\
0 & 0 & -a & 0 \\
0 & 0 & 0 & 1-2 a
\end{array}\right)
$$

with the linear depolarisation ratio $\delta$ and the polarisation parameter $a$ related by Eq. (4).

$$
\delta=\frac{F_{11}-F_{22}}{F_{11}+F_{22}} ; a=\frac{F_{11}}{F_{22}} \Rightarrow a=\frac{1-\delta}{1+\delta}
$$

\section{Standard measurements $\left(0^{\circ}\right)$}

The equations for the standard measurement intensities without calibrator have the form of Eq. (5) with the two new parameters $G_{S}$ and $H_{S}$.

$$
I_{S}=\eta_{S} T_{S} T_{O} T_{r o t} F_{11} T_{E} I_{L}\left(G_{S}+a H_{S}\right)
$$

The GH-parameters allow us to formulate general expressions for $\delta$ in Eq. (6)
$\delta=\frac{1-a}{1+a}=\frac{\delta^{*}\left(G_{T}+H_{T}\right)-\left(G_{R}+H_{R}\right)}{\left(G_{R}-H_{R}\right)-\delta^{*}\left(G_{T}-H_{T}\right)}$

with the calibrated signal ratio $\delta^{*}$ and the calibration factor $\eta$ in Eq. (7),

$\delta^{*}=\frac{1}{\eta} \frac{I_{R}}{I_{T}}\left(0^{\circ}\right) ; \quad \eta \equiv \frac{\eta_{R} T_{R}}{\eta_{T} T_{T}}$

and for the atmospheric backscatter coefficient $F_{11}$ in Eq. (8), which are valid for all lidar systems that can be described by the model in Fig. (1).

$F_{11} \propto \eta H_{R} I_{T}-H_{T} I_{R}$

The GH-parameters are fully described by the Stokes vector of the emitted laser beam $\boldsymbol{I}_{L}$ and the optical properties of the modules $\mathbf{M}_{E}, \mathbf{M}_{O}$, and $\mathbf{M}_{T, R}$ in Fig. 1, i.e. the diattenuation parameters $D$, the retardances $\Delta$, and the rotation $\theta$ of the modules around the optical axis.

\section{Calibration measurements $\left( \pm 45^{\circ}\right)$}

The calibration factor $\eta$ (Eq. (7)) has to be determined by means of measurements with additional calibration elements (calibrator) that should distribute the incident light in equal intensities to both channels ${ }_{T, R}$. For that purpose, four different calibrator types $\mathbf{C}$ can be inserted in our model at three locations as shown in Fig. 2.
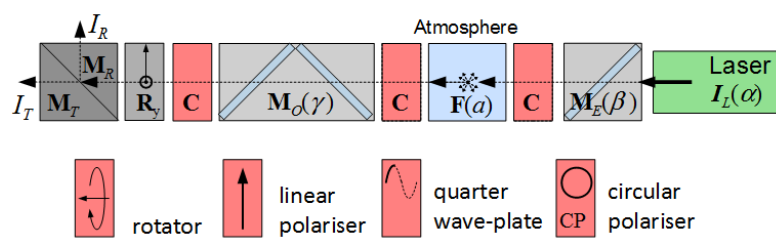

Figure 2 Different calibrator (red block) positions (top), and three options for the calibrator $\mathbf{C}$ (bottom).

We use as calibrator $\mathbf{C}$ the

- mechanical and $\lambda / 2$-plate rotation $\left(\mathbf{M}_{r o t}(\mathrm{~h})\right)$,

- a linear polariser $\left(\mathbf{M}_{P}\right)$,

- a $\lambda / 4$-plate $\left(\mathbf{M}_{Q W}\right)$, and

- a circular polariser $\left(\mathbf{M}_{C P}\right)$.

These calibrators should be oriented at + or $-45^{\circ}$ with respect to the plane of incidence of the PBS in order to distribute the incident light equally, but as the absolute $45^{\circ}$ orientation is difficult to achieve, an error $\varepsilon$ is included in Eq. (9) for the gain ratio $\eta^{*}$. The calibration signals $I_{S}$ in Eq. (9) include the same input Stokes vector and analyser 
part of Eq. (2) as the standard signals, but because of the different types of calibrators no general expression like Eq. (5) could be found for the calibration signals, and the expression for the calibration correction parameter $K$ (Eq. (9)) has to be determined for each lidar setup individually.

$\eta^{*}(\varepsilon)=\frac{I_{R}\left( \pm 45^{\circ}+\varepsilon\right)}{I_{T}\left( \pm 45^{\circ}+\varepsilon\right)}=K \eta$

It can be shown, that the combination of the $\pm 45^{\circ}$ measurements in Eq. (9) at exactly $90^{\circ}$ difference ( $\Delta 90$-calibration), as in Eq. (10), reduces the uncertainty of the calibration.

$$
\eta_{\Delta 90}^{*} \equiv \sqrt{\eta^{*}\left(+45^{\circ}+\varepsilon\right) \eta^{*}\left(-45^{\circ}+\varepsilon\right)}
$$

\section{Binary operators}

In order to further reduce the number of equations, we introduce three binary operators, $\mathrm{x}$, $\mathrm{h}$, and y. With $\mathrm{x}$ (Eq. (11)) we combine the equations for the $\pm 45^{\circ}$ calibration measurements. With operator $\mathrm{h}$ (Eq. (12)) we combine the calibration equations for the mechanical and the $\lambda / 2$-plate rotation $\left(\mathbf{M}_{r o t}(\mathrm{~h})\right)$. The $90^{\circ}$ rotation operator y (Eq. (13)) combines the equations for $90^{\circ}$-rotated PBSs (see Fig. (3)), because such a rotation is possible for some lidar systems.

$$
\begin{aligned}
& \mathrm{x}= \pm 1: \Psi(\mathrm{x}, \mathcal{E})=\mathrm{x} 45^{\circ}+\mathcal{E} \\
& \mathrm{h}= \pm 1: \mathbf{M}_{r o t}(\mathrm{x}, \mathcal{\varepsilon}, \mathrm{h})=\mathbf{R}\left(\mathrm{x} 45^{\circ}+\varepsilon\right) \mathbf{M}_{\mathrm{h}} \\
& \mathrm{y}= \pm 1: \boldsymbol{I}_{S}=\eta_{S} \mathbf{M}_{S} \mathbf{R}(\mathrm{y}) \mathbf{C} \mathbf{M}_{O} \mathbf{F} \mathbf{M}_{E} \boldsymbol{I}_{L}
\end{aligned}
$$

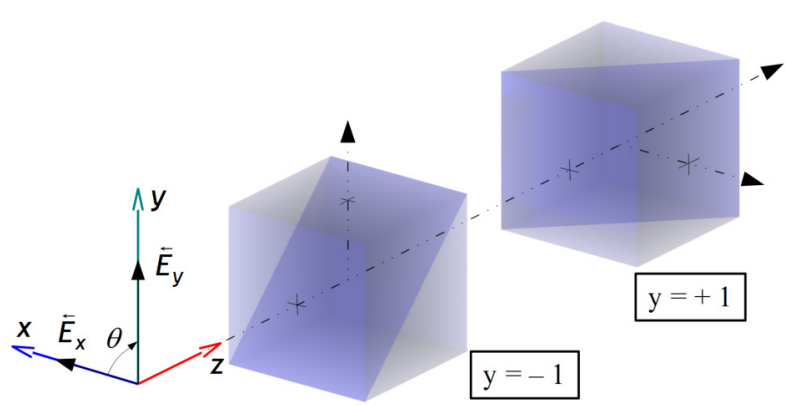

Figure 3 The orientation of the PBS with respect to the reference coordinate system for the two values of the binary operator y.

\section{BENEFITS}

The equations derived allow an analytical estimation of systematic error sources and their importance, and thus the optimization of the design of new and existing lidar systems with respect to their polarization properties. For example, it becomes evident that the $\Delta 90$ calibration largely removes errors due to a rotational misalignment $\varepsilon$ of the calibrator setup and that the cleaning of the cross talk of the PBS by means of additional polarization filters, which is very cheap and quite easy, simplifies the calibration equations, especially for the $\Delta 90$ calibration. Furthermore, we can directly compare the advantages and disadvantages of different calibrators at different locations and choose the best combination for our special lidar setup. With calibrators at different locations it is possible to determine the diattenuation of the receiving optics. With a $\lambda / 4$-plate calibrator it is possible to estimate the degree of circular polarization of the beam, and with a rotation-calibrator or a circular polarizing calibrator it is possible to determine the rotation of the plane of polarization of the laser beam.

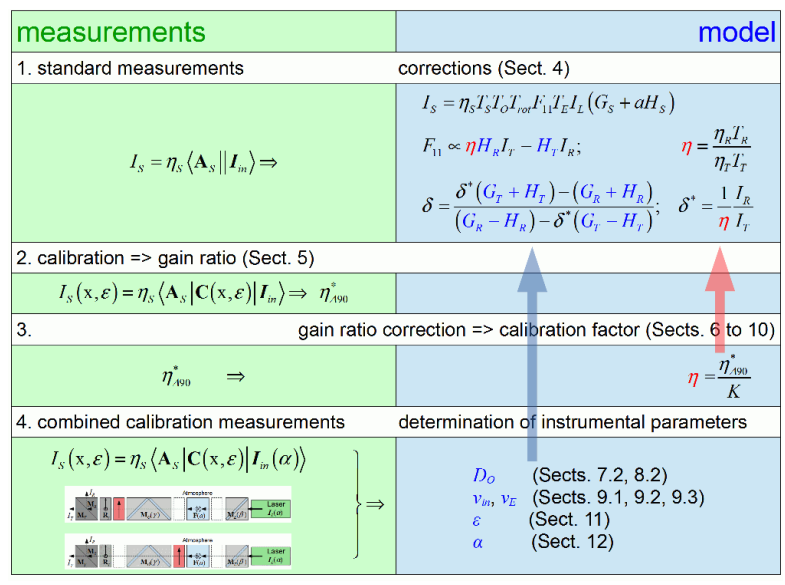

Figure 4 Four steps for calibrating and correcting the standard measurements for systematic errors by means of the model equations and additional calibration measurements. On the left side the measurements and their Müller matrix representations are listed and on the right side the scalar equations and their parameters, which can be retrieved from our model and from additional calibration measurements. References to sections are with respect to [1].

On the other hand, in case all optical parameters and rotational misalignments are known, it is possible to correct the measured signals with the 
provided equations. Fig. 4 summarizes the steps of this procedure.

In case the optical parameters and rotational misalignments are only known with uncertainties, a numerical complete search over the uncertainty space of all parameters (numerical error analysis) yields an overall systematic error estimation for the lidar signals and the retrieved linear depolarisation ratio and backscatter coefficient. Examples of such an error analysis for several EARLINET lidar systems with a Matlab code is shown by Bravo-Aranda et al. [3] (see their corresponding contribution at this conference), and an open source Python code including a real $\lambda / 2$-plate calibrator with retardation and other additional details has been published recently [4].

\section{ACKNOWLEDGEMENTS}

The financial support for EARLINET in the ACTRIS Research Infrastructure Project by the European Union's Horizon 2020 research and innovation programme under grant agreement

n. 654169 and previously under grant agreement n. 262254 in the $7^{\text {th }}$ Framework Programme (FP7/2007-2013) is gratefully acknowledged.

\section{References}

[1] Freudenthaler, V., 2016: About the effects of polarising optics on lidar signals and the 490 calibration, Atmos. Meas. Tech. 9, 4181-4255, doi: 10.5194/amt-9-4181-2016

[2] Mishchenko, M. I. \& Hovenier, J. W., 1995: Depolarization of light backscattered by randomly oriented nonspherical particles Opt. Lett., OSA, 20, 1356-1358, doi: 10.1364/OL.20.001356

[3] Bravo-Aranda, J., et al., 2016: Assessment of lidar depolarization uncertainty by means of Inewline a polarimetric lidar simulator, Atmos. Meas. Tech. 9, 4935-4953, doi: 10.5194/amt-94935-2016

[4] Freudenthaler, V., 2017: Open source Python code for polarization related error analysis of aerosol lidar signals:

https://bitbucket.org/iannis_b/atmospheric_lidar_ghk 